Article

\title{
An evaluation of social media images portrayal of disability discourse: \#amputeefitness
}

\author{
Fallon R. Mitchell ${ }^{*}$, Sara Santarossa1, Isuri L. Ramawickrama1, Emily F. Rankin'1, Jessica A. \\ Yaciuk $^{1}$, Erin R. McMahon ${ }^{1}$ and Paula M. van Wyk ${ }^{1}$
}

Received: $9^{\text {th }}$ September, 2018; Accepted: $5^{\text {th }}$ August 2019; Published: $6^{\text {th }}$ October 2019

\begin{abstract}
The widespread use and influential impact of social media has made it increasingly important to analyze the social norms and discourses that are being presented and promoted among social media users. Thus, the purpose of this study was to examine whether the conversation and images associated with \#amputeefitness on Instagram promoted the medical model discourse (i.e., a focus on aesthetic) or the social model of disability discourse (i.e., a focus on adapted physical activity). Using the Netlytic software, all publicly available Instagram media tagged with \#amputeefitness were collected, and a text and image analysis were performed. The text analysis revealed that the conversation was positive or neutral and focused on four themes: community, motivation, aesthetic, and physical activity. The image analysis suggested an emphasis was placed on body structure (i.e., having an acceptable appearance) and physical activity. Although there was evidence of the medical model within the posts associated with \#amputeefitness, there was a stronger indication of a shift towards the social model of disability discourse.
\end{abstract}

Keywords: social model of disability; medical model; body image; Instagram; physical activity; Netlytic

\section{Introduction}

Affecting 1.6 million people in the United States (Christensen, Ipsen, Doherty, \& Langberg, 2016) and an unknown incidence within Canada (Imam, Miller, Finlayson, Eng, \& Jarus, 2017), there are several types of amputations caused by a variety of factors. The two primary categories of amputations are congenital and acquired. Congenital amputations result from the failure of a limb to properly form, and often do not have an established cause (Jain \& Lakhtakia, 2002); whereas, acquired amputations are commonly the result of trauma, vascular conditions, inflammation, and cancer (Bragaru, Dekker, Geertzen, \& Dijkstra, 2011). From a physical standpoint, individuals with congenital amputations and individuals with acquired amputations may be indistinguishable from one another. However, from a psychological standpoint, they often differ significantly (Pepper \& Willick, 2009). Individuals with congenital amputations typically have the body image and selfidentity of an individual without a disability, whereas individuals who have an acquired amputation experience greater shock, anxiety, depression, and anger, after limb loss occurs (Pepper \& Willick, 2009). Thus, an individual who experiences an acquired amputation may face greater challenges in adapting and renegotiating their identity in several life domains including physical appearance, functional limitations, body image, quality of life, and social support (Senra, Oliveira, Leal, \& Vieira, 2012). However, physical activity, inclusive of exercise, sport, and leisure activities, may assist in reducing these challenges (Pepper \& Willick, 2009). By improving an individual's physical fitness, satisfaction with appearance, self-confidence, coping abilities, and overall quality of life, physical activity can play a key role in rehabilitation, and additionally aid individuals to live independently, have improved body image, increased opportunities for participation, and facilitate improved social interactions (Bragaru et al., 2011; Pepper \& Willick, 2009; Wetterhahn Hanson \& Levy, 2002). Thus, physical activity may be employed as a tool to foster social acceptance, promote inclusion 
(Tatar, 2010), and can be used to shape an individual's beliefs regarding many aspects of their life, including body image and self-identity (van Amsterdam, Knoppers, \& Jongmans, 2015).

In general, physical activity can afford individuals an improved psychological wellbeing (Hausenblas \& Fallon, 2006). However, society has created an aesthetic ideal that emphasizes vitality, physical appearance, and fitness, from which any deviation of this ideal may be seen as a failure, thus, contributing to a negative body image (Holzer et al, 2014). This appears to be an embedded viewpoint that dates back to ancient Greece in which the ideal individual is described as being in harmony with the universe, or perfect equilibrium, unaffected by old age and disease, and that any deviations were a god-sent punishment (Tountas, 2009). During this time period, male bodies were viewed as having the potential to be the ideal specimen and that women were a mutilated or deformed version of men (Horowitz, 1976). Although these may not be the exact views of the male and female body today, there are still these ideals that everyone should uphold or be subjected to ridicule such as body shaming. For example, the media primarily depicts females with thin and toned bodies (Carrotte, Prichard, \& Lim, 2017; Tiggemann \& Zaccardo, 2015) and males with muscular bodies (Carrotte et al., 2017), emphasizing appearance-related benefits, such as thinness and muscularity, thus, further contributing to body image distortion (Tiggemann \& Zaccardo, 2015). Individuals may compare themselves to the unattainable bodies illustrated in fitness images, fostering a negative body image (Tiggemann \& Zaccardo, 2015). Although, they may contribute to a negative body image (Tiggemann \& Zaccardo, 2015), fitness images also have the ability to inspire individuals to engage in active lifestyles (Carrotte et al., 2017; Tiggemann \& Zaccardo, 2015). Therefore, it is increasingly important to examine images that display diverse body types, which may improve body image, and inspire physical activity.

Within disability research there are many perspectives from which a variety of models and theories have emerged, including those of critical disability studies, the medical model, the social model of disability, and more. Critical disability studies builds upon and questions traditional, dominant theories to challenge and reformulate understandings of disability (Goodley, 2013; Meekosha \& Shuttleworth, 2009; Vehmas \& Watson, 2014). The theoretical basis for critical disability studies is multidisciplinary incorporating aspects of ableism, phenomenology, intersectionality, and feminist theories. Critical disability studies attempt to break down the binary understanding of disability (i.e., able-bodied and disabled), which acts as a normative constraint by suggesting that there is a 'right' and 'wrong' ability or body (Meekosha \& Shuttleworth, 2009; Vehmas \& Watson, 2014). Furthermore, critical disability studies aim to shift away from the dominant disablism perspective and its negative connotations, to a more positive ableism perspective (Vehmas \& Watson, 2014). Critical disability studies extend beyond the biological and materialistic approaches of the medical model and social model of disability to incorporate the fluidity of identity formation and representation, cultural meanings, and diversity (Goodley, 2013; Vehmas \& Watson, 2014). Thus, critical disability studies postulates that disability does not simply result from an individual's biological properties (i.e., medical model) or material barriers within society (i.e., social model), rather disability acts as a social body that is largely dependent on corporeal, societal, and cultural factors (Goodley, 2013; Vehmas \& Watson, 2014).

Although there are multiple disability discourses, models and lenses, this study will focus on the medical model and the social model of disability, as these are (currently) the dominant models within society (Goodley, 2013). From a medical model standpoint, the mind and body are considered separate entities where the body is approached as a collection of parts and subjective experiences are regarded as irrelevant (Turner, 2001). In line with the medical model, barriers are viewed as a personal misfortune, resulting from the individual's impairment (Brittain, 2004; Stamou, Alevriadou, \& Soufla, 2016). Thus, emphasizing discrimination and rehabilitating individuals, if possible, back to 'normal' standards (Brittain, 2004; Stamou et al., 2016). From the perspective of the medical model, individuals with an amputation deviate from the 'normal' and 'ideal' body as they have a disfigurement that needs to be fixed or hidden. In contrast to having individuals of a perceived elite status (e.g., physician) defining disability, the social model of disability was conceptualized by individuals who have a disability (Lang, 2001) that consider the body to be socially produced (Turner, 
2001) and view barriers as a social (including environment), political, and cultural construct (Stamou et al., 2016). In accordance with this latter model, the 'ideal' body is a barrier to positive body image constructed by society, and that individuals with an amputation could achieve the 'ideal' body. While the medical model was the previously dominant standard in society, as a result of the elitist and exclusionary approach to defining disability, there has been a shift towards the social model of disability (Lang, 2001). This shift affords individuals with a disability the discourse and system to perceive their bodies in a positive light, and overcome other barriers they may face (i.e., social exclusion, loss of independence).

Although advancements have been made towards the social model of disability, there is still movement that needs to be made, and the media could play a key role in this progression. Historically, the media has portrayed individuals with an amputation as a symbol of evil by depicting a frightening persona among certain characters (Dahl, 1993). As a result, society may be influenced to believe that individuals with a disability, and in particular an amputation, should be feared (Bandura, 2001). This was demonstrated in the story Peter Pan with the classic villain, Captain Hook, who was intentionally depicted as an individual with a prosthetic; a silver hook in place of his hand (Dahl, 1993). By seeing characters react to individuals with an amputation in a negative way, said reactions could be internalized by the audience (Bandura, 2001; Hogg, Terry, \& White, 1995). As a result, observers may transfer negative reactions towards individuals with an amputation into reality (Bandura, 2001). Furthermore, individuals with an amputation may internalize the notion that they are unappealing, which negatively impacts their self-efficacy as this notion conflicts with their self-perception (Bandura, 2001; Hogg et al., 1995). This decline in self-efficacy, needs to be combatted, as efficacy, in general, influences an individual's motivation, commitment to goals, and physical and mental well-being (Bandura, 2001).

Traditional media (e.g., television, newspapers, and magazines), has contributed to the development of three dominant representations of disability: supercrips, disadvantaged, or ill victims (Zhang \& Haller, 2013). Although, the supercrip is a more positive representation of disability than the historic symbol of evil (Zhang \& Haller, 2013), it has received mixed reactions (Silva \& Howe, 2012). Perhaps one of the best examples of the supercrip ideology is seen in media coverage of the Paralympic Games. Paralympians are portrayed as having functional, productive, and efficient bodies that challenge the limitations of the 'disabled' body by demonstrating impressive accomplishments (Silva \& Howe, 2012). While some suggest that supercrips may cause individuals with a disability to set unattainable goals and feel inadequate if they cannot achieve supercrips status (Quinlan \& Bates, 2008), others suggest that this representation may instill confidence in individuals with a disability (Zhang \& Haller, 2013). By seeing individuals with similar characteristics (e.g., a disability) to themselves obtain great accomplishments, individuals with a disability may experience an improved self-identity (Zhang \& Haller, 2013).

In contrast, the latter two representations, that is disadvantaged or ill victims, have negative implications and reinforce the stigmatization of disability (Zhang \& Haller, 2013). Charity advertising is one domain in which the disadvantaged and victimized representations of disability are exploited (Barnes \& Mercer, 2001). Through the emphasis of an individual's physical disability, charity advertising attempts to generate donations by eliciting feelings of fear or sympathy in viewers (Barnes \& Mercer, 2001). Representing individuals with a disability as disadvantaged, helpless, and/or victims in the media may cause individuals with a disability to develop low self-esteem and feel inferior to able-bodied individuals (Zhang \& Haller, 2013). However, one avenue that individuals may use to share or even redefine their identity is through social media, where individuals can create an online profile, share multimedia content, and connect with other users (Bowles, 2016; Boyd \& Ellison, 2007; Senra et al., 2012). Since media has the potential to alter the way individuals think and behave through demonstration (i.e., images) and description (i.e., captions and comments), it could prove to be essential in changing social practices and behaviour patterns (Bandura, 2001). In particular, social media can communicate messages on a global scale, which could be beneficial in the promotion of physical activity among individuals with a disability (Bowles, 2016) and the development of a community or social network. 
With the growing use of social media, body image, and physical activity have become a primary research focus among images on social networking sites (e.g., Facebook, Twitter, and Instagram; Perloff, 2014). However, there has been a paucity of research that has examined these images as they relate to individuals with a disability, and in particular, an amputation. Recently, research has focused on the examination of hashtags that related to body image and physical activity, such as \#fitspo (Carrotte et al., 2017; Santarossa, Coyne, Lisinski, \& Woodruff, 2016). Hashtags can be used by individuals on social media who identify as being in the same social category (e.g., female, Canadian, athlete) to create a community (Ashforth \& Mael, 1989; Bowles, 2016). For example, the hashtag \#amputeefitness is used on Instagram, a social networking site that shares visual content and has gained popularity since its launch in 2010 (Bowles, 2016), to foster a community among individuals with an amputation. The purpose of this study was to examine whether the conversation and images associated with \#amputeefitness promoted a personal aesthetic (i.e., medical model discourse with respect to the need to hide or fix an individual's amputation to achieve the 'ideal body') or demonstrated adapted physical activity (i.e., social model of disability discourse by promoting inclusion, adaptations, and overcoming social and environmental barriers).

\section{Methods}

Using the Netlytic program (Gruzd, 2016), images on Instagram tagged with \#amputeefitness were collected, a text analysis was conducted, and an image coding scheme was developed by the research team and implemented for \#amputeefitness images. By providing the hashtag (\#amputeefitness) that was analyzed for this study the FAIR principles have been addressed, as the data are searchable and publicly accessible through a search of \#amputeefitness images that are dated between January 13th and January 20th, 2018. As noted throughout the paper, all images used in this study were publicly available. Each method is further discussed below.

\section{Data collection and Netlytic analysis}

The Netlytic program (Gruzd, 2016), is an open-sourced software, and was used to download all publicly available media from Instagram tagged with \#amputeefitness. The hashtag \#amputeefitness (more than 11,000 posts as of March 2019) was selected based on its use on Instagram in comparison to similar options such as \#fitamputee (868 posts) or \#amputeefitfam (5 posts). Thus, \#amputeefitness was selected for analysis, as it was thought that having more tagged posts would ensure the generation of 50 unique, author only images during data collection. The data were collected in real time for seven days, beginning on January 13th, 2018 and ending on January 20th, 2018. The Netlytic program (Gruzd, 2016) created an excel output file that recorded the image link, publication date, author of the hashtag (i.e., who wrote \#amputeefitness, which was not always the same as who posted the picture), the actual comment containing \#amputeefitness, geographical location, to whom the post was directed (if applicable), the number of 'likes' the image received, and if an Instagram filter was used on the image. Additionally, the Netlytic program (Gruzd, 2016) collected, organized (by frequency), and filtered (i.e., removed filler words, such as 'the', or 'and') words or hashtags found within the discussion on Instagram. Of the 2951 words or hashtags, the research team chose to analyze the top 30 terms, as has been previously performed in the literature (Santarossa et al., 2016). This was based on the number of messages (i.e., the number of messages the term appeared in) and instances (i.e., the number of times the term appeared), as well as the identification of common themes.

\section{Image selection}

The excel file ( $\mathrm{N}=809$ records), generated by the Netlytic program (Gruzd, 2016), was downloaded and 50 unique author only, topic related images were randomly sampled for photo coding analysis. The research team chose to analyze 50 images, as manual coding of the images was performed, and 50 images was deemed reasonably manageable. Prior to the selection of 50 images for analysis, the data were cleaned for author only images (i.e., the author of the image who had used \#amputeefitness in the caption of their image), unique images (no repeated images), unique authors 
(no repeated authors), and any videos, deleted images/accounts, or inappropriate images were excluded from further analysis.

\section{Coding of images}

This study used a novel image coding scheme to analyze images tagged with \#amputeefitness. The coding scheme that was developed was based on adapted physical activity (APA) terms, as described by the International Classification of Functioning, Health and Disability (ICF) (World Health Organization [WHO], 2001), and Sport Science categories, which were discussed by Hutzler (2010), adapted from Hutzler and Sherrill (2007). Although the ICF is more heavily weighted towards the medical model, it was designed to encompass medical and social model perspectives. Additionally, as there was no pre-existing coding scheme, both APA and ICF were selected as the basis to guide the development of a coding scheme for this study. The ICF was divided into overarching categories, one of which was 'functioning and disability', which was further divided into four main interrelated subcategories: body functions, body structures, activities, and participation (WHO, 2013). In addition to the ICF, APA was developed to encourage and support accepting attitudes, advocating access to active lifestyles and sport, and promoting empowerment for individuals with a disability (International Federation of Adapted Physical Activity [IFAPA], 2014).

The content categories within the code book specified the type of sport or physical activity the image depicted, the number of people in the image, if there was a service provider such as a physiotherapist or coach in the image, if the focus of the image was of an athlete, non-athlete or scenery, as well as the type of shot (e.g., a selfie, headshot, half body, full body, or other). An image could be coded for multiple ICF categories, as these categories were not exclusive of each other. For example, an image coded for body structure could, also, be coded for participation in physical activity. For each of these content categories, the images were further coded into subcategories based on the ICF category guidelines. For example, the variable 'Body Function', which was described as the physiological functions of body systems, may have had images that were further classified as being able to perform (e.g., restoring range of motion) which included subcategory coding options for aerobic and anaerobic endurance, muscle strength and endurance, joint flexibility, psychological traits, and energy efficiency and power. An overall code for each category was determined by majority consensus, and any discrepancies were resolved by coming to an agreement among the members of the research team.

\section{Results}

\section{Data collection and Netlytic analysis}

Among the 809 records downloaded, the 30 most frequently used, unique, meaningful words or hashtags associated with \#amupteefitness were analyzed. The most frequent word or hashtag was \#amputeefitness, and thus was not included as it was the topic of this study. Therefore, among the 30 most frequent words or hashtags associated with \#amputeefitness, \#amputee, which had 131 messages and 131 instances, became the top word or hashtag. Of the words or hashtags considered for text analysis, $17.0 \%$ were words $(n=5)$, and $83.0 \%$ were hashtags $(n=25)$. A sample of the most commonly used words or hashtags, amassed by Netlytic (Gruzd, 2016) to be associated with \#amputeefitness can be found in Table 1 .

These 30 most common words or hashtags were manually sorted into common themes by the researchers (Figure 1), including: community (e.g., \#fitfam and \#amputee), motivation (e.g., great and love), aesthetic (e.g., back and \#amputeestyle), and physical activity (e.g., \#amputeeswholift and \#gymnastics). The majority of words or hashtags related to the theme 'community' ( $n=11 ; 37.0 \%)$ and 'motivation' $(n=9 ; 33.0 \%)$, whereas the lesser two themes that emerged were physical activity $(n=6$; $20.0 \%)$ and aesthetic $(n=3 ; 10.0 \%)$. 
Table 1. Most commonly used words or hashtags associated with \#amputeefitness.

\begin{tabular}{ccc}
\hline Term & Number of messages & Number of instances \\
\hline \#amputee & 131 & 131 \\
\#amputeelife & 79 & 82 \\
\#gym & 59 & 59 \\
\#amputeeswholift & 56 & 57 \\
\#fitness & 56 & 57 \\
\#amputeestrong & 49 & 49 \\
\#amputees & 40 & 40 \\
\#amputeepride & 38 & 38 \\
great & 35 & 37 \\
\#fitfam & 34 & 34 \\
\#gymmotivation & 30 & 30 \\
\#amputeeathlete & 28 & 28 \\
\#amputeemodel & 28 & 28 \\
\#gymlife & 27 & 27 \\
work & 26 & 31 \\
\hline
\end{tabular}

\section{Themes}

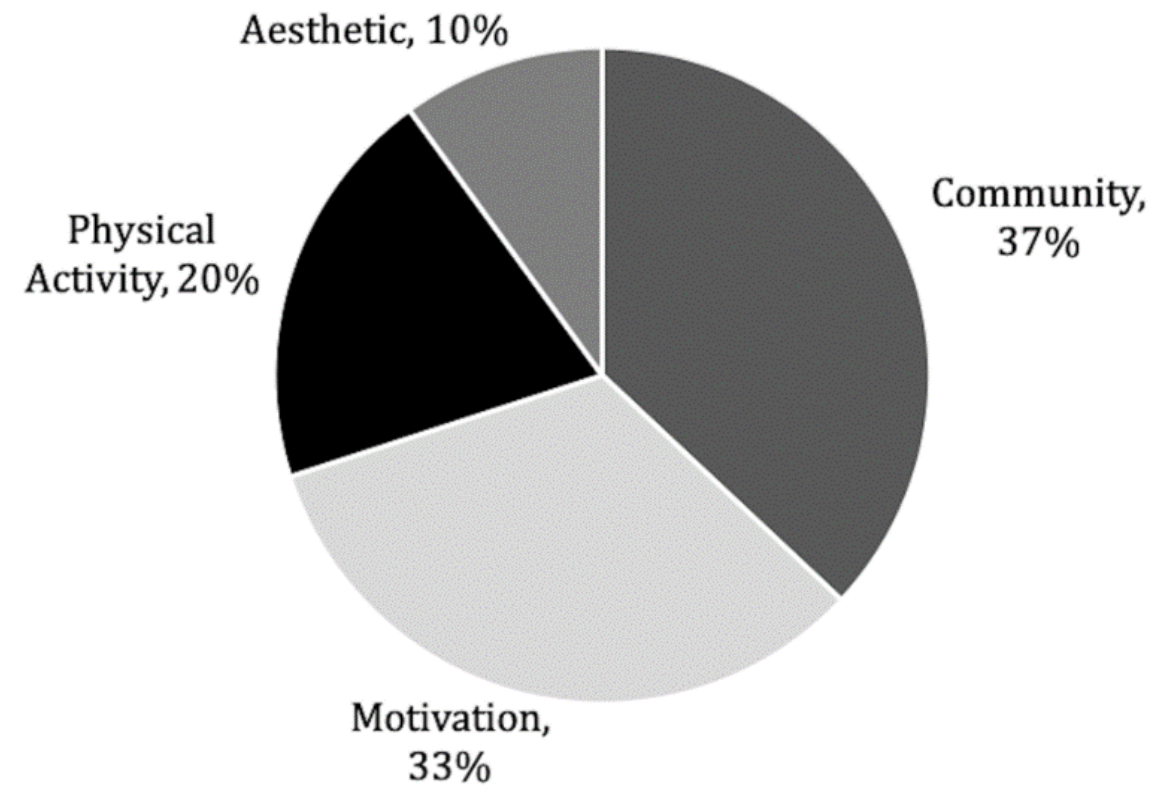

Figure 1. Categories of popular words or hashtags associated with \#amputeefitness ( $n=30)$.

\section{Image selection and coding of images}

The intent of this study was to collect images over a seven-day period using the Netlytic (Gruzd, 2016) software to collect 50 unique, topic relevant images produced by unique authors. After the 809 records were downloaded, they were sorted by unique image $(n=185)$ and then by unique author $(n$ $=49$ ), and further cleaned for content, with only 34 images remaining eligible for coding (Figure 2). Of the 34 images, 18 images (53.0\%) pertained to physical activity or sport, and demonstrated strength training, swimming, rock climbing, running, soccer, or stretching. While 16 images $(47.0 \%)$ did not relate to physical activity or sport, they did demonstrate individuals posing, modelling, and inanimate objects, such as prosthetic devices, membership cards, screenshots, protein powder, or food. The number of people in the images ranged from zero to three, with a total of 26 images $(77.0 \%)$ 
including people, and eight images with no people. From the 26 images that included people, 19 images $(56.0 \%)$ had one person, five images had two people $(15.0 \%)$, and two images had three people $(6.0 \%)$. Of the images that included people, two images $(6.0 \%)$ had a service provider present, such as a physiotherapist or coach. The majority of images $(n=18 ; 53.0 \%)$ focused on an athlete or physical activity participant as the subject of the image, while the remaining images $(n=16 ; 47.0 \%)$ focused on nonathletes, family members, friends, or other. The type of shot varied for the 34 images, with the majority $(n=20 ; 59.0 \%)$ being full body shots.

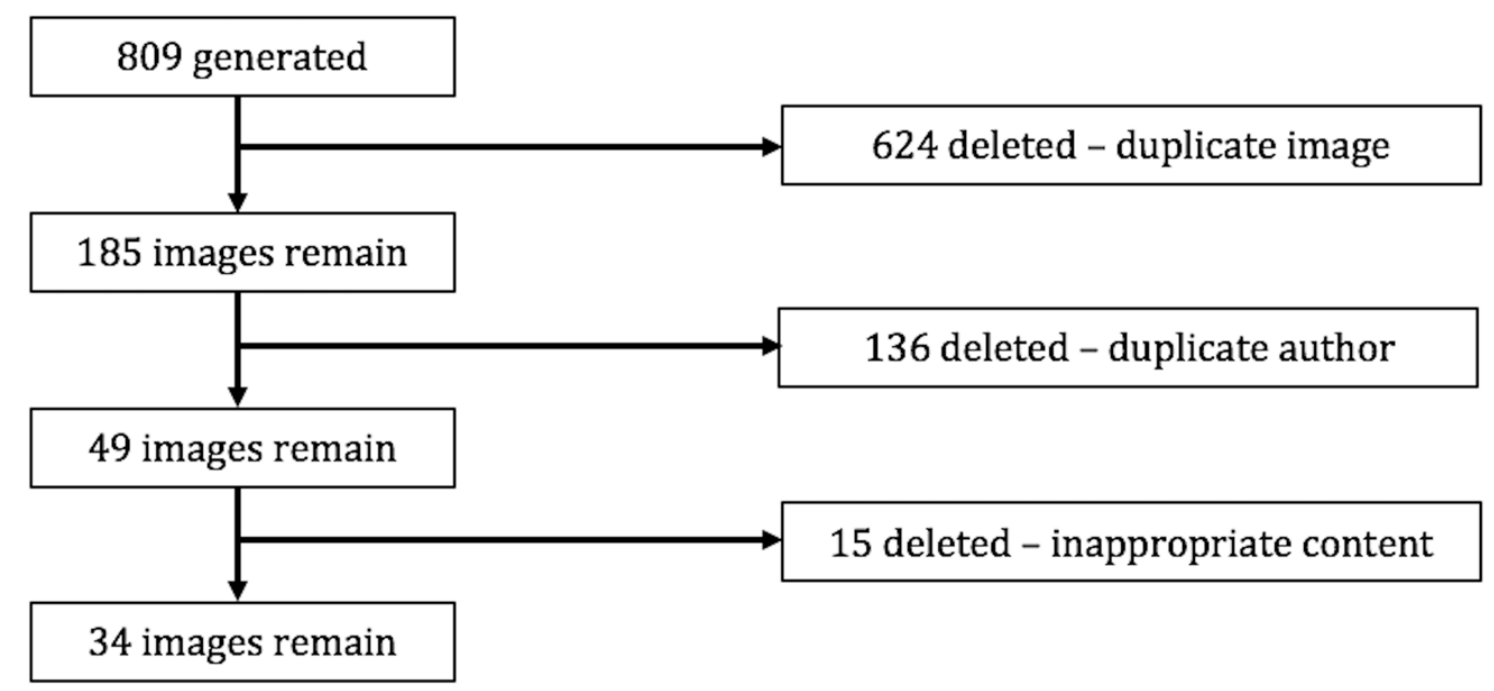

Figure 2. Inclusion tree for images tagged with \#amputeefitness and reasons for exclusion from analysis.

The ICF categories that were used to analyze the images were body structure, body function, activity or task performance, participation in physical activity, and elimination of barriers to goal achievement. Body structure (68.0\%), the only category to exceed $50.0 \%$ of the images coded, was the largest ICF category, and participation in physical activity (23.5\%) was the smallest category (Figure 3).

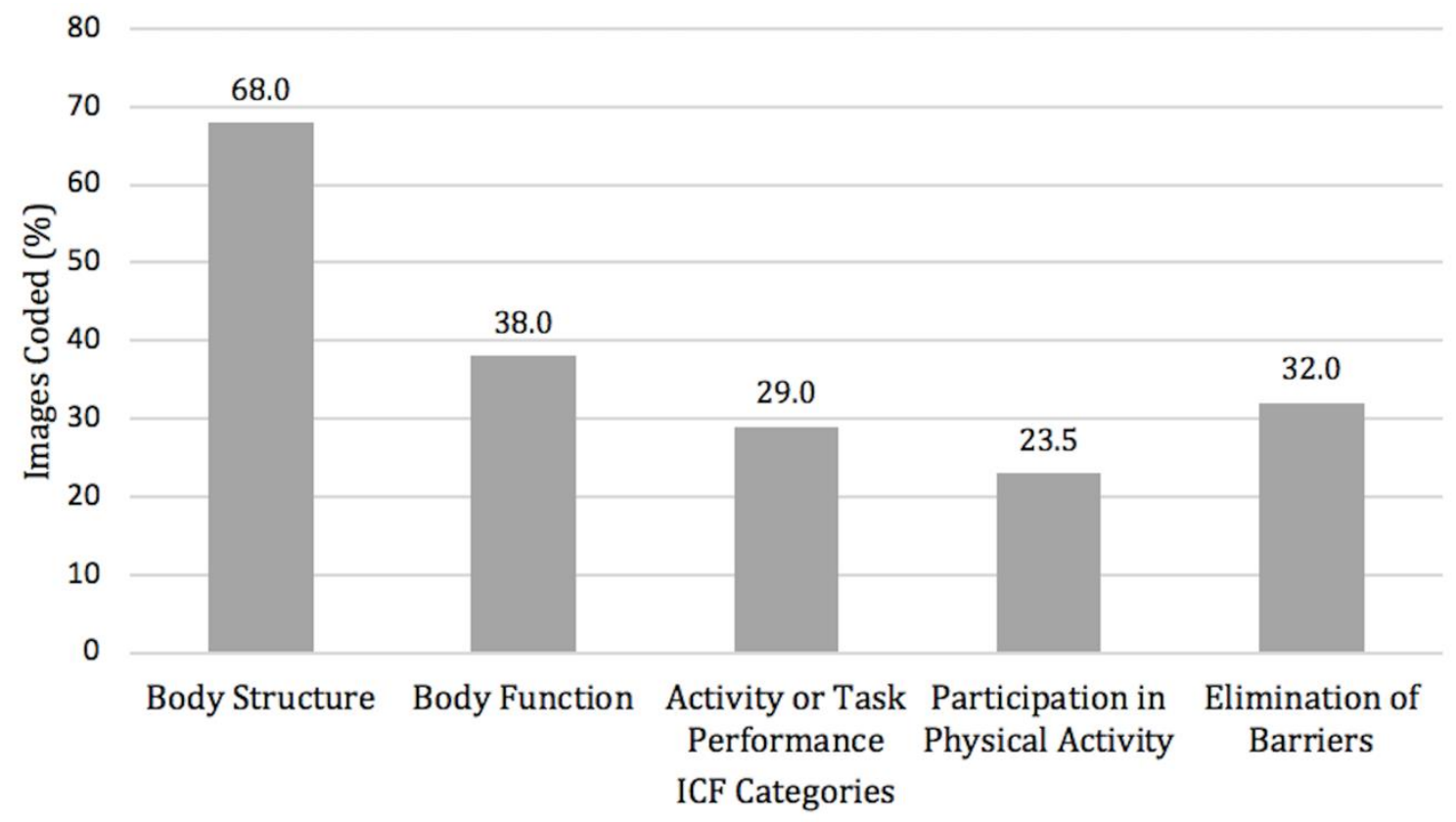

Figure 3. Percent of images coded for each International Classification of Functioning, Health and Disability (ICF) category. 
The images that focused on body structure emphasized either physical foundation, having an acceptable appearance, or both. These 23 images were further sub-categorized into neuromuscular and muscles $(\mathrm{n}=14 ; 61.0 \%)$, aesthetic $(\mathrm{n}=8 ; 35.0 \%)$, and cardiorespiratory and respiratory $(\mathrm{n}=1$; $4.0 \%)$. However, there were no images coded for the neurological sub-category.

Thirteen images (38.0\%) focused on body function, as they represented the ability to perform, with $69.0 \%(n=9)$ further coded for muscle strength and endurance, $15.0 \%(n=2)$ for aerobic and anaerobic endurance (including lung and cardiovascular function), one (8.0\%) for joint flexibility, and one $(8.0 \%)$ for energy efficiency and power. No images were further coded for psychological traits.

For the ICF category activity or task performance, ten of the images (29.0\%) focused on activity or task performance in relation to physical activity, and illustrated the participant doing a meaningful task such as training to improve balance and strength, swimming for an hour, running, or rock climbing. Within this category, all $(100.0 \%)$ focused on sport-specific skills, as opposed to fundamental motor or psychological skills. Twenty-four images (71.0\%) within the ICF category activity or task performance, did not focus on the performance of meaningful tasks, rather, they depicted posing, modelling, or inanimate objects.

Additionally, within the ICF category elimination of barriers, only $32.0 \%$ of images $(n=11)$ depicted adaptations, of which, $82.0 \%(n=9)$ of the images depicted individuals demonstrating adaptations using equipment, such as prosthetic or assistive devices, $18.0 \%(n=2)$ demonstrating adaptations through social support systems, such as personal trainers or foundations, and no images demonstrating adaptations through advocacy, rules, or environments.

Other aspects of the images that were coded for included overall photo type (e.g., posed shots, athletic action, or other), passive/active, and clothing. In regard to the overall photo type, half of the images $(n=17 ; 50.0 \%)$ were considered posed shots, with $65.0 \%$ of those images $(n=11)$ having no athletic marker, and $35.0 \%(n=6)$ having an athletic marker, such as athletic equipment. Furthermore, nine images $(26.5 \%)$ were categorized as other, and eight images were athletic action shots $(23.5 \%)$. Half of the images $(n=17 ; 50.0 \%)$ were considered to be passive non-sport, while seven images $(20.6 \%)$ were passive in sport, seven images $(20.6 \%)$ were active in sport, and three images $(8.8 \%)$ were active non-sport. Of all 34 images, $28(82.0 \%)$ were categorized as unrevealing, as the individuals wore casual gym or physical activity clothing, four images $(12.0 \%)$ were slightly revealing, and two images $(6.0 \%)$ had individuals wearing a bathing suit, lingerie, or a sports bra and bottoms. The average number of likes on the images coded were 78 , and 22 images $(65.0 \%)$ did not have a filter, while 12 images $(35.0 \%)$ did have a filter. Of the images with a filter, the most popular was 'Clarendon' with three images (25.0\%) using this filter.

\section{Discussion}

This study examined if the conversation and images tagged with \#amputeefitness on Instagram promoted personal aesthetic (i.e., medical model discourse) or adapted physical activity (i.e., social model of disability discourse). Based on 30 words or hashtags and 34 eligible images, the results were broken into a text and image analysis. Although the medical model has been the traditionally accepted viewpoint in disability discourse, and striving to depict an 'ideal' body image at first seemed to provide evidence that the data in this study supported the notion that this model was still prevalent, further critical analysis of this finding began to illustrate potential support for the social model of disability. Additional findings from the data, such as formulating a sense of community and promoting participation in (adapted) physical activity, also supported the shift towards endorsing the social model of disability.

\section{Aesthetic}

Contrary to the notion that aesthetic (i.e., an emphasis on physical appearance) would most closely align with the medical model discourse, in which images may depict individuals with an amputation hiding their 'disfigurement' or 'fixing' their amputation to reflect a 'normal' physical appearance, the findings of this study indicated that aesthetic could represent both models. As the majority of coded images focused on body structure, which was described to emphasize the 
promotion of an acceptable appearance with respect to physical foundation (Hutzler, 2010), there was evidence to support a medical model presence. Striving to emulate the 'ideal' body in which females have been typically associated with thin, toned bodies (Carrotte et al., 2017; Tiggemann \& Zaccardo, 2015) and males have been perceived as muscular (Carrotte et al., 2017), it is not surprising the Instagram posts tagged with \#amputeefitness were also imitating these features. Furthering the potential alignment with the medical model, individuals with an amputation may have felt the need to hide their amputation as a missing limb deviates from the prescribed ideal body (Holzer et al., 2014; Tiggemann \& Zaccardo, 2015). Evidence for this hiding of the amputation may be provided by noting that several \#amputeefitness posts did not visibly display the so-called deviation from an 'ideal' body (e.g., amputation). Although it is possible that users felt societal pressures to conform to an ideal image, this perspective of needing to fit a mould that would be viewed and interpreted by others as a 'normal' body aligns with the medical model. If this was the case, the perceived need for an individual to hide their amputation because it deviates from the 'ideal' body may foster a negative body image (Holzer et al., 2014; Tiggemann \& Zaccardo, 2015) and introduce challenges to successful social reintegration (Wetterhahn et al., 2002). However, it may have been an unintentional oversight that images excluded an evident and visible amputation. Although a large portion of images were perceived as emphasizing the ideals associated with body structure and thus that of the medical model, the use of \#amputeefitness within the posts provided a direct label that highlighted what one may assume was to be hidden (i.e., their amputation). Thus, it may otherwise be noted that focusing on aesthetic could suggest that individuals using \#amputeefitness were confident, had a positive body image, and felt individuals with an amputation could achieve the aesthetic ideal, thus, representing the social model of disability.

In addition to the explicit tagging of posts with \#amputeefitness, thus, potentially promoting the social model of disability, there were a paucity of negative words or hashtags in the text analysis generating a conversation that was interpreted as positive (e.g., great) or neutral (e.g., \#amputeestyle). Eliminating negativity from the conversation may dissociate individuals with an amputation from negative emotions (e.g., fear), positively changing the pervasive image that surrounds amputations (Bandura, 2001; Hogg et al., 1995) or, in general, tones that may be more directly aligned with the medical model discourse. A more positive perception of amputations may create an environment in which it could be internalized that individuals with an amputation can achieve an aesthetic ideal, thus, improving the self-efficacy of individuals with an amputation (Bandura, 2001; Hogg et al., 1995) and providing further support for the transition towards a social model of disability discourse. Furthermore, there were images that depicted modelling, or posing and that made the amputation visible, potentially providing support for the indication that individuals with an amputation may have developed self-efficacy, positive body image, and felt they could achieve the aesthetic ideal. Expanding the aesthetic ideal to include diverse bodies, such as those with an amputation, could derive positive body image for all who do not fit the thin and toned, or muscular 'ideals' by promoting more authentic bodies on Instagram. In other words, although the images may have been depicting aspects of body structure, such as muscularity, the intent may not have been to depict an ideal image, but to align with the social model of disability by complying with a population that is otherwise viewed as displaying ideal and desirable characteristics.

\section{Community}

The words or hashtags used within the posts to create a positive or neutral conversation, coupled with direct labelling (through the use of \#amputeefitness), may be indicating that users were formulating a sense of community among each other. The theme of community first emerged through hashtags, such as \#amputee and \#fitfam, which were interpreted as relating to a strong connection of a communal or familial presence. Hashtags are one way in which Instagram may aide in establishing communities (Saxton, Niyirora, Guo, \& Waters, 2015) by enabling individuals to connect based on common interests and similar characteristics (Veale et al, 2015). As the text analysis in the present study was primarily comprised of hashtags, it was believed that users were attempting to create connections that foster an online community (Saxton et al., 2015; Veale et al., 2015). The use of 
multiple hashtags (e.g., \#amputeefitness, \#amputee, and \#fitfam) further suggested that users were attempting to create multiple online communities and expand their reach to individuals perceived to be similar, whether these individuals also have an amputation or a passion for fitness.

One of the connections that appeared evident through the fostering of an online community was the connection to fitness or physical activity. For example, \#fitfam enabled individuals, both with and without an amputation, to connect and interact based on a shared interest in fitness. As \#fitfam was not specific to individuals with an amputation, the use of this hashtag suggested that individuals with an amputation were comparable to, and assimilate with, a medical model characterization of able-bodied individuals. Thus, by using hashtags to formulate a sense of community among individuals who either had an amputation or a passion for fitness, users were reinforcing the social model of disability by breaking down barriers and creating avenues for online connections. Removing this social barrier, that is viewing an amputation as a deviation that ostracizes individuals from being accepted by others, could enhance communication and a sense of belonging between individuals with an amputation among like individuals and communities of traditionally labelled able-bodied individuals. The removal of social barriers may decrease social isolation, increase social support, and improve self-confidence, all of which may be necessary for obtaining a positive body image and link back to an overall confident aesthetic (Bragaru et al., 2011). Just as striving to depict an ideal body image or aesthetic and formulating a sense of community promoted the social model of disability discourse, promoting participation in physical activity represented the social model of disability discourse through text and images that appeared to illustrate adaptations, and overcoming the barriers of society and the environment.

\section{Physical Activity and Motivation}

Hashtags in the text analysis, such as \#amputeeswholift and \#gymnastics, clearly indicated physical activity as a key part of the conversation surrounding \#amputeefitness posts. Traditionally, physical activity was thought to be an activity in which individuals with an amputation should not participate, as it would aggravate their 'condition', that is their amputation (Brittain, 2004), thus, highlighting the medical model discourse and the need to be rehabilitated. However, the appearance of physical activity in the conversation surrounding \#amputeefitness may further indicate a shift towards the social model of disability discourse, as it suggests that physical activity is a topic in which individuals with an amputation can be (and should be) included. Physical activity may be an avenue through which individuals can advocate for and promote inclusive and adaptive environments, by overcoming social and environmental barriers. As a focal component of social model of disability discourse, the environment is a barrier that needs to be overcome (Brittain, 2004). Data interpreted from this study suggests that \#amputeefitness images that illustrated equipment modifications and body function (i.e., the ability to perform) demonstrated a shift towards the social model of disability through the elimination of environmental barriers. Images that depicted equipment modification were demonstrating, whether intentional or not, the capabilities of individuals with an amputation to lead a physically active lifestyle. The promotion of their capabilities further contradicts the notion that individuals with an amputation should not or cannot participate in physical activity, thus, enabling individuals with diverse bodies and unique needs to participate in physical activity and potentially creating a more inclusive environment. Consequently, the publicly available \#amputeefitness images may help to foster and inspire a wider variety of individuals to partake in more active lifestyles, which may lead to increased fitness among individuals with a disability potentially resulting in improved body function and more positive body image (Charalampos, Silva, \& Kudlacek, 2015).

Similar to the manner in which 'fitspiration' images (i.e., images that aim to inspire healthier lifestyles and physical activity) may motivate individuals to engage in healthy eating, exercise, and self-care by emphasizing strength and empowerment (Tiggemann \& Zaccardo, 2015), \#amputeefitness may motivate individuals to engage in physical activity, by emphasizing the capabilities of individuals with an amputation. Further, improved fitness, body image, and body shape have been found to be motivational factors for participation in sport (Charalampos et al., 2015). Thus, the 
promotion of aesthetic demonstrated within 'fitspiration' imagery may encourage individuals with an amputation to increase their physical activity. Although further investigation is required, it is possible that the motivational theme that emerged from the text analysis may further support that fitness images can motivate participation in physical activity. A more in-depth text analysis should be conducted to determine the context of the motivation (i.e., whether or not motivation was being used to encourage an aesthetic ideal or physical activity).

However, the illustration of individuals' capabilities could be mistaken for 'cripspiration' (i.e., individuals with a disability being portrayed as heroic for completing otherwise everyday activities; Liddiard, 2014). This misconception may be a result of the media often portraying individuals with amputations, who participate in physical activity, as 'cripspiration' (Dahl, 1993; Liddiard, 2014). For example, the athlete Terry Fox, was portrayed in the media as a hero for his accomplishments (Dahl, 1993). Terry Fox was a 22-year-old Canadian who attempted to run across Canada to raise money for the fight against cancer with an amputated right leg, a resultant from cancer (The Terry Fox Foundation, 2018). Rather than discussing the environmental conditions that led to Terry Fox's cancer and subsequently his amputation, the media emphasized Terry Fox as the 'dying' hero, who overcame his amputation and achieved heroic accomplishments (Dahl, 1993). As 'cripspiration' emphasizes an individual's disability as a barrier to overcome, 'cripspiration' also reinforces the medical model and is potentially demotivating (Liddiard, 2014). However, Instagram produces usergenerated media (Shao, 2009), providing individuals the opportunity to frame their posts in a way of their choosing, for example emphasizing the social model of disability. By showing adaptations (e.g., equipment modification) that permitted participation in physical activity, \#amputeefitness images were able to highlight the environmental barriers that they had to overcome, demonstrating that their disability was not the barrier, thus, aligning more with motivation than 'cripspiration'.

Additionally, the perspective of the social model of disability indicates that social barriers need to be overcome (Brittain, 2004). The use of social media, in addition to the depiction of social support systems in these images, demonstrated the elimination of social barriers, thus, further indicating the presence of the social model of disability. While social media and social support systems may provide human support, social media also serves to break down the barriers of face-to-face communication, further reducing social barriers, thereby creating communities (Santarossa, Kane, Senn, \& Woodruff, 2018). The abilities enabled through the use of social media to enact behaviour change (Santarossa et al., 2018; Veale et al., 2015), alter social norms (Veale et al., 2015), and create supportive and accepting communities (Shao, 2009; Veale et al., 2015) could increase participation in physical activity. Thus, the combination of social support with participation in physical activity could lead to the development of communities that encourage active lifestyles. When individuals with an amputation interact with a physically active community online (or offline) and receive support and acceptance, they may be motivated to engage in more active lifestyles, potentially aiding in the reduction of antisocial effects often associated with physical disability (Charalampos et al., 2015). An increase in physical activity could result in improved or restored body image (Tatar, 2010), as physical activity may improve their perceived level of fitness (Wetterhahn et al., 2002). Improved body image leads to improved self-confidence, which can contribute to improved social interactions (Charalampos et al., 2015). As an individual's body image improves, so does their ability to reintegrate with society (Pepper \& Willick, 2009). Thus, the emergence of community, motivation, aesthetic, and physical activity in the text analysis may indicate that \#amputeefitness was creating a community that motivated individuals to participate in physical activity and realize they can (and do) have an 'ideal' body.

\section{Limitations}

The Instagram posts associated with \#amputeefitness were collected over a span of one week. In doing a quick search for \#fitfam, over 91 million posts (August 27th, 2018) were available to view publicly. In searching a few other hashtags that appeared in the most frequently used text with the images of this study the following post numbers were found: \#gymlife over 40 million; \#gymmotivation almost 10.5 million; \#amputeestrong approximately 16.5 thousand; \#amputeeswholift one more than 13.5 
thousand; and \#amputeefitness just greater than 8 thousand. A similar search for \#amputeefitness was performed prior to data collection. Thus, it was originally assumed that this would be a feasible amount of time to attain 50 unique image and unique author posts. However, this was not the case as there were only 34 images included for analysis after the data were cleaned. Perhaps a search spanning longer than one week would have generated more images for inclusion in this study. However, the inability to generate 50 usable unique author unique images draws attention to why that situation may arise. In reviewing the original 809 records, 624 were duplicate images. This may be a result of users seeing an image elsewhere and deciding to also post it on their Instagram page. There were also 136 images excluded as they were from a duplicate author. Thus, although the interpretation of the data may suggest that there is a growing community to support inclusion and physical activity online among individuals with an amputation, the reach of these individuals may currently not be large. A shift towards a social model of disability discourse is viewed to be favourable (Brittain, 2004), but there are many barriers that still exist. The two other hashtags (\#amputeestrong and \#amputeeswholift) had more posts, but they were also arguably using terms associated with body structure (e.g. muscularity) and potentially may be illustrating a medical model culture. However, as with the body structure images of this study, it is also possible that these images were still depicting a social model of disability discourse; further analysis is needed. Regardless, it is evident that there were fewer Instagram posts associated with amputation than fitness or physical activity in general. The presence of the medical model demonstrated by the results of this study may be partially attributable to the use of the ICF as the basis for the code book. The ICF, although designed to be a combination of the medical model and social model of disability, more heavily favours medical model perspectives (Imrie, 2004). However, the authors felt as though using the ICF as the basis of the code book would better reflect aspects of both models, rather than alienating a specific model.

The idiom "a picture is worth a thousand words" highlights that what the authors of this study perceived to be portrayed in the \#amputeefitness Instagram images may differ from the perceptions of other viewers, and may also differ from the intention of the user who made the posts. Future research should conduct a more in-depth text analysis, as this could provide context to the themes that emerge, helping to understand the thoughts and feelings of those posting the images and those leaving comments. For example, although motivation emerged as a theme, the context (i.e., what users were being motivated to do) of the users' motivation was unknown. Additionally, it may be advantageous for a future study to survey or interview Instagram users who have posted images using \#amputeefitness to directly ask about their intentions.

Furthermore, it cannot be determined by viewing an image whether or not an individual had a congenital or acquired amputation. As individuals with congenital amputations may have significantly different psychological experiences than individuals with acquired amputations (Pepper \& Willick, 2009) the images and results may be interpreted differently based on the type of amputation.

\section{Conclusions}

Overall, this study indicated that there is a shift towards the social model of disability discourse among Instagram users who used \#amputeefitness. Although assimilating to the 'ideal' body may indicate the continued presence of the medical model, promoting a more diverse aesthetic ideal, creating a sense of community, and encouraging physical activity were interpreted as promoting the social model of disability discourse. The apparent acceptance of the social model of disability, supported by the evidence of this study, is necessary for individuals with a disability to be able to participate in physical activity, reintegrate with society, and to recognize that society is the barrier, rather than an individual's impairments (Lang, 2001). As Instagram gives users the power to generate their own media and reach large audiences, it becomes increasingly important to further explore the norms and ideals that users are promoting and representing online.

\section{Perspectives}


The implications of this study could be applied in the promotion of rehabilitation programs, by understanding the effects these programs could have on an individual's well-being (i.e., physical capabilities, body image, confidence, social interaction, and motivation), if they begin to incorporate social media. Due to the paucity of negative tones associated with \#amputeefitness, the ideology that social media is used to establish a positive community can be applied to promote and establish physical activity programs. Furthermore, physical activity programs should be more accessible to individuals with a disability, by having a more adaptive and inclusive environment, which could be accomplished by providing adapted equipment, and supportive and knowledgeable staff. Understanding the ways in which each model is represented on social media may assist in changing the dominant discourse to that of the social model of disability. For example, individuals should be encouraged to participate in physical activity and embrace their amputation, not hide it. Additionally, future interventions aimed at improving body image should emphasize that there is not a single 'ideal' body, rather, there are many. Diversity in body types should be encouraged, as it is not the individual's body, but what is considered 'ideal' that needs to change.

\section{Author affiliations:}

1 University of Windsor, Department of Kinesiology; mitch11e@uwindsor.ca, santaros@uwindsor.ca, ramawici@uwindsor.ca, ranki114@uwindsor.ca, yaciukj@uwindsor.ca, mcmahone@uwindsor.ca, pvanwyk@uwindsor.ca

* Correspondence: mitch11e@uwindsor.ca; Tel.: 519-253-3000

Author Contributions: Conceptualization, F.R.M., S.S. and P.M.V.W.; Methodology, S.S. and P.M.V.W.; Formal Analysis, F.R.M., S.S., I.L.R., E.F.R., J.A.Y. and E.R.M..; Writing-Original Draft Preparation, F.R.M., S.S., I.L.R., E.F.R., J.A.Y., E.R.M., and P.M.V.W.; Writing-Review \& Editing, F.R.M., S.S. and P.M.V.W.

Funding: This research received no external funding

Conflicts of Interest: The authors declare no conflict of interest.

\section{References}

Ashforth, B. E., \& Mael, F. (1989). Social identity theory and the organization. Academy of Management Review, 14, 20-39. doi:10.5465/amr.1989.4278999

Bandura, A. (2001). Social cognitive theory of mass communication. Media Psychology, 3, 265-299. doi:10.1207/S1532785XMEP0303_03

Barnes, C., \& Mercer, G. (2001). Disability culture: Assimilation or Inclusion? Handbook of disability studies. doi:10.4135/9781412976251.n22

Bowles, J. (2016). Instagram: A visual view of the southeastern conference. Journal of Contemporary Athletics, 10 227-238.

Boyd, D., \& Ellison, N. (2007). Social network sites: Definition, history, and scholarship. Journal of ComputerMediated Communication, 13, 210-230. doi:10.1111/j.1083-6101.2007.00393.x

Bragaru, M., Dekker, R., Geertzen, J. H., \& Dijkstra, P.U. (2011). Amputees and sports. Sports Medicine, 41, 721740. doi:10.2165/11590420-000000000-00000

Brittain, I. (2004). Perceptions of disability and their impact upon involvement in sport for people with disabilities at all levels. Journal of Sport \& Social Issues, 28, 429-452. doi:10.1177/0193723504268729

Carrotte, E. R., Prichard, I., \& Lim, M. S. C. (2017). “Fitspiration” on social media: A content analysis of gendered images. Journal of Medical Internet Research, 19, e95. doi:10.2196/jmir.6368

Charalampos, S., Silva, C. F., \& Kudlacek, M. (2015). When sitting becomes sport: Life stories in sitting volleyball. European Journal of Adapted Physical Activity, 8, 30-44. doi:10.5507/euj.2015.003

Christensen, J., Ipsen, T., Doherty, P., \& Langberg, H. (2016). Physical and social factors determining quality of life for veterans with lower-limb amputation(s): A systematic review. Disability and Rehabilitation, 38, 23452353. doi:10.3109/09638288.2015.1129446

Dahl, M. (1993). The role of the media in promoting images of disability-disability as metaphor: The evil crip. Canadian Journal of Communication, 18, 75-80. doi:10.22230/cjc.1993v18n1a718

Goodley, D. (2013). Dis/entangling critical disability studies. Disability \& Society, 28, 631-644. doi:10.1080/09687599.2012.717884

Gruzd, A. (2016). Netlytic: Software for automated text and social network analysis. Retrieved from http://Netlytic.org 
Hausenblas, H. A., \& Fallon, E. A. (2006). Exercise and body image: A meta-analysis. Psychology and Health, 21, 33-47. doi:10.1080/14768320500105270

Hogg, M. A., Terry, D. J., \& White, K. M. (1995). A tale of two theories: A critical comparison of identity theory with social identity theory. Social Psychology Quarterly, 58, 255-269. doi:10.2307/2787127

Holzer, L. A., Sevelda, F., Fraberger, G., Bluder, O., Kickinger, W., \& Holzer, G. (2014). Body image and selfesteem in lower-limb amputees. PLoS One, 9, e92943. doi:10.1371/journal.pone.0092943

Horowitz, M. C. (1976). Aristotle and woman. Journal of the History of Biology, 9, 183-213. doi:10.1007/bf00209881

Hutzler, Y. (2010). International Encyclopedia of Rehabilitation. Buffalo, NY: Center for International Rehabilitation Research Information and Exchange (CIRRIE).

Hutzler, Y., \& Sherrill, C. (2007). Defining adapted physical activity: International perspectives. Adapted Physical Activity Quarterly, 24(1), 1-20. doi:10.1123/apaq.24.1.1

Imam, B., Miller, W. C., Finlayson, H. C., Eng, J. J., \& Jarus, T. (2017). Incidence of lower limb amputation in Canada. Canadian Journal of Public Health, 108, 374-380. doi:10.17269/cjph.108.6093

Imrie, R. (2004). Demystifying disability: A review of the international classification of functioning, disability and health. Sociology of Health E Illness, 26, 287-305. doi:10.1111/j.1467-9566.2004.00391.x

International Federation of Adapted Physical Activity (IFAPA). (2014). Definition of Adapted Physical Activity [Webpage]. Retrieved from http://ifapa.net/definition/

Jain, S., \& Lakhtakia, P.K. (2002). Profile of congenital transverse deficiencies among cases of congenital orthopaedic anomalies. Journal of Orthopaedic Surgery, 10, 45-52. doi:10.1177/230949900201000109

Lang, R. (2001). The development and critique of the social model of disability. London: Leonard Cheshire Disability and Inclusive Development Centre.

Liddiard, K. (2014). Media review: Liking for like's sake- the commodification of disability on Facebook. Journal on Developmental Disabilities, 20, 94-101.

Meekosha, H., \& Shuttleworth, R. (2009). What's so 'critical'about critical disability studies? Australian Journal of Human Rights, 15, 47-75. doi:10.1080/1323238X.2009.11910861

Pepper, M., \& Willick, S. (2009). Maximizing physical activity in athletes with amputations. Current Sports Medicine Reports, 8(6)339-344. doi:10.1249/JSR.0b013e3181c1db12

Perloff, R. (2014). Social media effects on young women's body image concerns: Theoretical perspectives and an agenda for research. Sex Roles, 71, 363-377. doi:10.1007/s11199-014-0384-6

Quinlan, M. M., \& Bates, B. R. (2008) Dances and discourses of (Dis)ability: Heather Mills's embodiment of disability on dancing with the stars. Text and Performance Quarterly, 28, 64-80. doi:10.1080/10462930701754325

Santarossa, S., Coyne, P., Lisinski, C., \& Woodruff, S. J. (2016). \#fitspo on Instagram: A mixed-methods approach using Netlytic and photo analysis, uncovering the online discussion and author/image characteristics. Journal of Health Psychology, 1-10. doi:10.1177/1359105316676334

Santarossa, S., Kane, D., Senn, C. Y., \& Woodruff, S. J. (2018). Exploring the role of in-person components for online health behavior change interventions: Can a digital person-to-person component suffice? Journal of Medical Internet Research, 20. doi:10.2196/jmir.8480

Saxton, G. D., Niyirora, J. N., Guo, C., \& Waters, R. D. (2015). \#AdvocatingForChange: The strategic use of hashtags in social media advocacy. Advances in Social Work, 16,1, 154-169. doi:10.18060/17952

Senra, H., Oliveira, R., Leal, I., \& Vieira, C. (2012). Beyond the body image: A qualitative study on how adults experience lower limb amputation. Clinical Rehabilitation, 26,2, 180-19. doi:10.1177/0269215511410731

Shao, G. (2009). Understanding the appeal of user-generated media: A uses and gratification perspective. Internet Research, 19, 7-25. doi:10.1108/10662240910927795

Silva, C. F., \& Howe, P. D. (2012). The (in) validity of supercrip representation of Paralympian athletes. Journal of Sport and Social Issues, 36, 174-194. doi:10.1177/0193723511433865

Stamou, A., Alevriadou, A., \& Soufla, F. (2016). Representations of disability from the perspective of people with disabilities and their families: A critical discourse analysis of disability groups on Facebook. Scandinavian Journal of Disability Research, 18,1, 1-16. doi:10.1080/15017419.2014.962611

Tatar, Y. (2010). Body image and its relationship with exercise and sports in Turkish lower-limb amputees who use prosthesis. Science $\mathcal{E}$ Sports, 25, 312-317. doi:10.1016/j.scispo.2010.02.001

The Terry Fox Foundation. (2018). The Marathon of Hope [Webpage]. Retrieved from http://www.terryfox.org/terrys-story/marathon-of-hope/

Tiggemann, M., \& Zaccardo, M. (2015). "Exercise to be fit, not skinny": The effect of fitspiration imagery on women's body image. Body Image, 15, 61-67. doi:10.1016/j.bodyim.2015.06.003

Tountas, Y. (2009). The historical origins of the basic concepts of health promotion and education: The role of ancient Greek philosophy and medicine. Health Promotion International, 24, 185-192. doi:10.1093/heapro/dap006 
Turner, B. S. (2001). Disability and the sociology of the body. In G.L. Albrecht, K. Sleeman, \& M. Bury (Eds.), Handbook of disability studies, (pp. 252-266). doi:10.4135/9781412976251.n10

van Amsterdam, N., Knoppers, A., \& Jongmans, M. (2015). 'It's actually very normal that I'm different'. How physically disabled youth discursively construct and position their body/self. Sport, Education and Society, 20, 152-170. doi:10.1080/13573322.2012.749784

Veale, H. J., Sacks-Davis, R., Weaver, E. R., Pedrana, A. E., Stoové, M. A., \& Hellard, M. E. (2015). The use of social networking platforms for sexual health promotion: Identifying key strategies for successful user engagement. BMC Public Health, 15,1, 1-11. doi:10.1186/s12889-015-1396-z

Vehmas, S., \& Watson, N. (2014). Moral wrongs, disadvantages, and disability: a critique of critical disability studies. Disability \& Society, 29,4, 638-650. doi:10.1080/09687599.2013.831751

Wetterhahn, K. A., Hanson, C., \& Levy, C. E. (2002). Effect of participation in physical activity on body image of amputees. American Journal of Physical Medicine \& Rehabilitation, 81,3, 194-201. doi:10.1097/00002060200203000-00007

World Health Organization (WHO). (2001). The International Classification of Functioning, Disability, and Health (ICF). Geneva: WHO Publications.

World Health Organization (WHO). (2013). How to use the ICF: A Practical Manual for using the International Classification of Functioning, Disability and Health (ICF). Geneva: WHO Publications.

Zhang, L., \& Haller, B. (2013). Consuming image: How mass media impact the identity of people with disabilities. Communication Quarterly, 61, 319-334. doi:10.1080/01463373.2013.776988

(C) 2019 by the authors. Submitted for possible open access publication under the terms and conditions of the Creative Commons Attribution (CC BY) license (http://creativecommons.org/licenses/by/4.0/). 